\title{
Sondage de l'Association des médecins princi- paux d'hôpitaux de Suisse (AMPHS): affiliation à la FMH à titre d'organisation de base?
}

Hans-Ueli Wuersten, président de l'AMPHS

Thomas Eichenberger, directeur de l'AMPHS

\section{Médecins-cadres en Suisse}

L'Association des médecins principaux d'hôpitaux de Suisse (AMPHS) englobe les médecinscadres en fonction dirigeante dans les hôpitaux suisses (médecins-chefs et médecins adjoints). L'association est divisée en sections cantonales qui délèguent en proportion de leurs effectifs un certain nombre de membres dans l'organe décisionnel supérieur (assemblée des délégués).

Nous estimons que les médecins en Suisse qui occupent une fonction dirigeante sont entre 2500 et 3000 . Il s'agit principalement de médecins engagés auprès d'hôpitaux (le plus souvent publics) dans une fonction de formateurs et de prestateurs de soins dans le cadre de l'assurance sociale. Les médecins d'hôpitaux salariés dans une fonction dirigeante peuvent aussi désormais devenir membres de l'AMPHS s'ils sont engagés dans des hôpitaux privés avec ou sans mandat de formation ou de prestations.

Selon l'art. 20 des statuts de la FMH, l'AMPHS représente les intérêts des médecins-chefs et médecins adjoints travaillant en milieu hospitalier face à la population, aux autorités et aux autres institutions. L'AMPHS comprend actuellement près de 1000 membres.

\section{Position au sein de la FMH}

L'AMPHS est représentée par un siège au Comité central de la FMH. Au sein de la Chambre médicale, l'AMPHS n'a que la fonction et le poids d'une société de discipline médicale (et y est représentée par deux délégués).

Sa représentation lacunaire, par rapport aux tâches précitées, au sein de l'instrument décisionnel supérieur de la FMH, a incité l'AMPHS à présenter deux fois de suite, dans un court intervalle, une demande de reconnaissance en tant qu'organisation de base. Cette proposition a été rejetée par la Chambre médicale, quoique de manière moins évidente la deuxième fois.
Un mandat demeure à l'étude, celui donné par la Chambre médicale d'examiner l'éventualité d'une fusion avec l'ASMAC sous la forme d'une organisation faîtière des médecins d'hôpitaux. Les travaux sont en cours et un rapport devrait être présenté à ce sujet à la prochaine Chambre médicale.

\section{Développements}

Le problème récurrent demeure, celui de la représentation lacunaire de l'AMPHS au sein de la Chambre médicale, tout particulièrement le manque d'identification des médecins-cadres avec les sociétés médicales cantonales en tant qu'organisations de base auxquelles ils doivent adhérer.

Les sociétés cantonales de médecine n'ont jusqu'ici pas répondu ou de manière trop vague aux propositions constructives de l'AMPHS. On pourrait aussi songer à faire de l'AMPHS une organisation de base tout en sauvegardant l'affiliation obligatoire dans les sociétés cantonales. Les cotisations pourraient être fractionnées en conséquence.

Les circonstances étant ce qu'elles sont, il est à craindre que les médecins-cadres pourraient décider, dans certains cantons du moins, de sortir de la société médicale cantonale.

\section{Enquête}

\section{Enquête concernant l'AMPHS en tant} qu'organisation de base

En été 2003, l'AMPHS a mené une enquête auprès de tous les médecins-cadres en Suisse. Sans être un franc succès, le taux de réponses a été satisfaisant. Pour 2750 questionnaires envoyés, on compte ainsi 804 participants qui ont renvoyé la formule assortie de leurs commentaires. Le taux de réaction a été ainsi de d'env. 29\%, ou d'un tiers. Près de la moitié des participants ne sont pas membres de l'AMPHS (368). Plus de la moitié en font partie (436). d'hôpitaux de Suisse (AMPHS)

Kapellenstrasse 14

CH-3011 Berne 
Figure 1

Enquête concernant l'AMPHS en tant qu'organisation de base de la FMH.

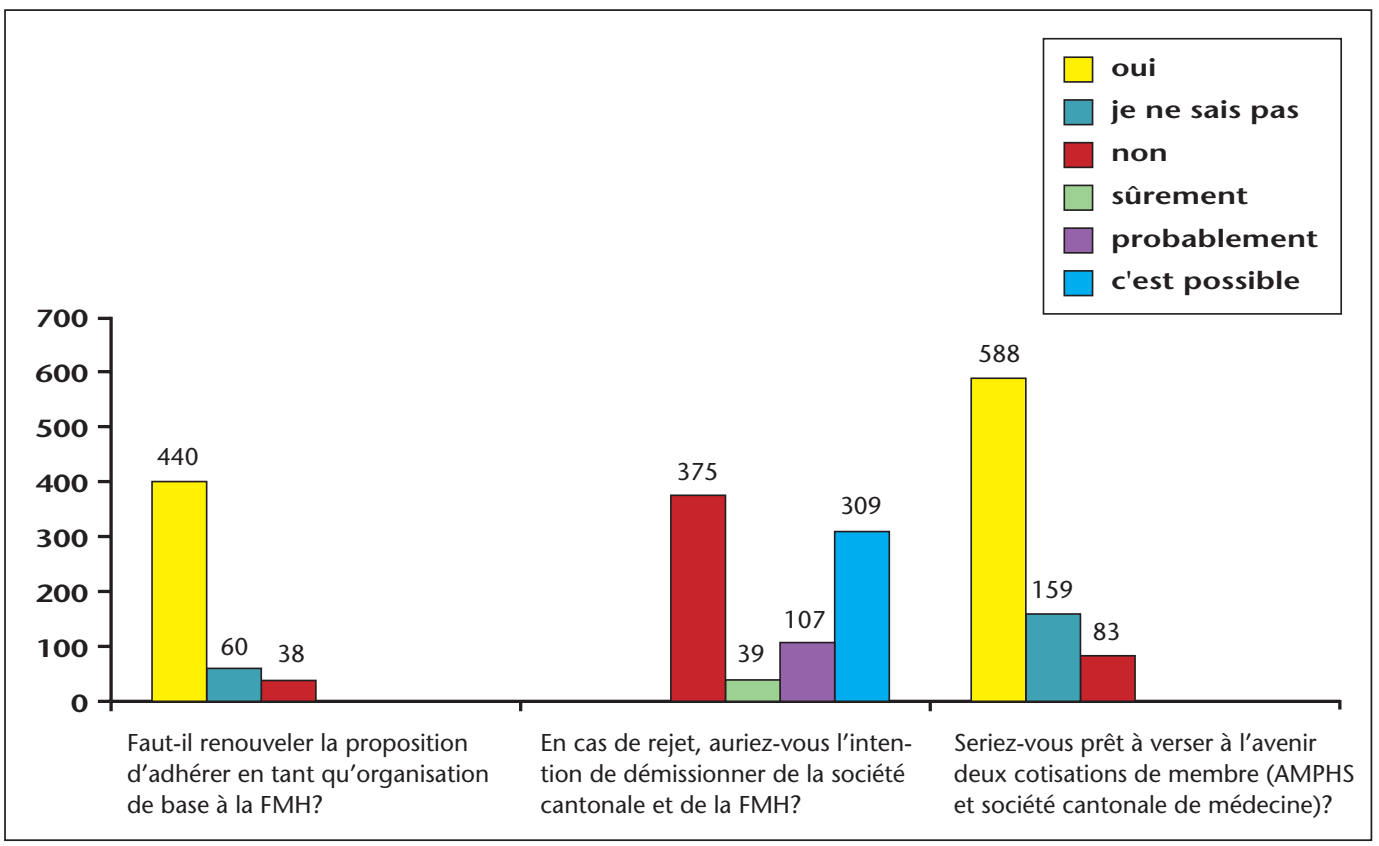

Figure 2

Enquête concernant les activités et les prestations de l'AMPHS.

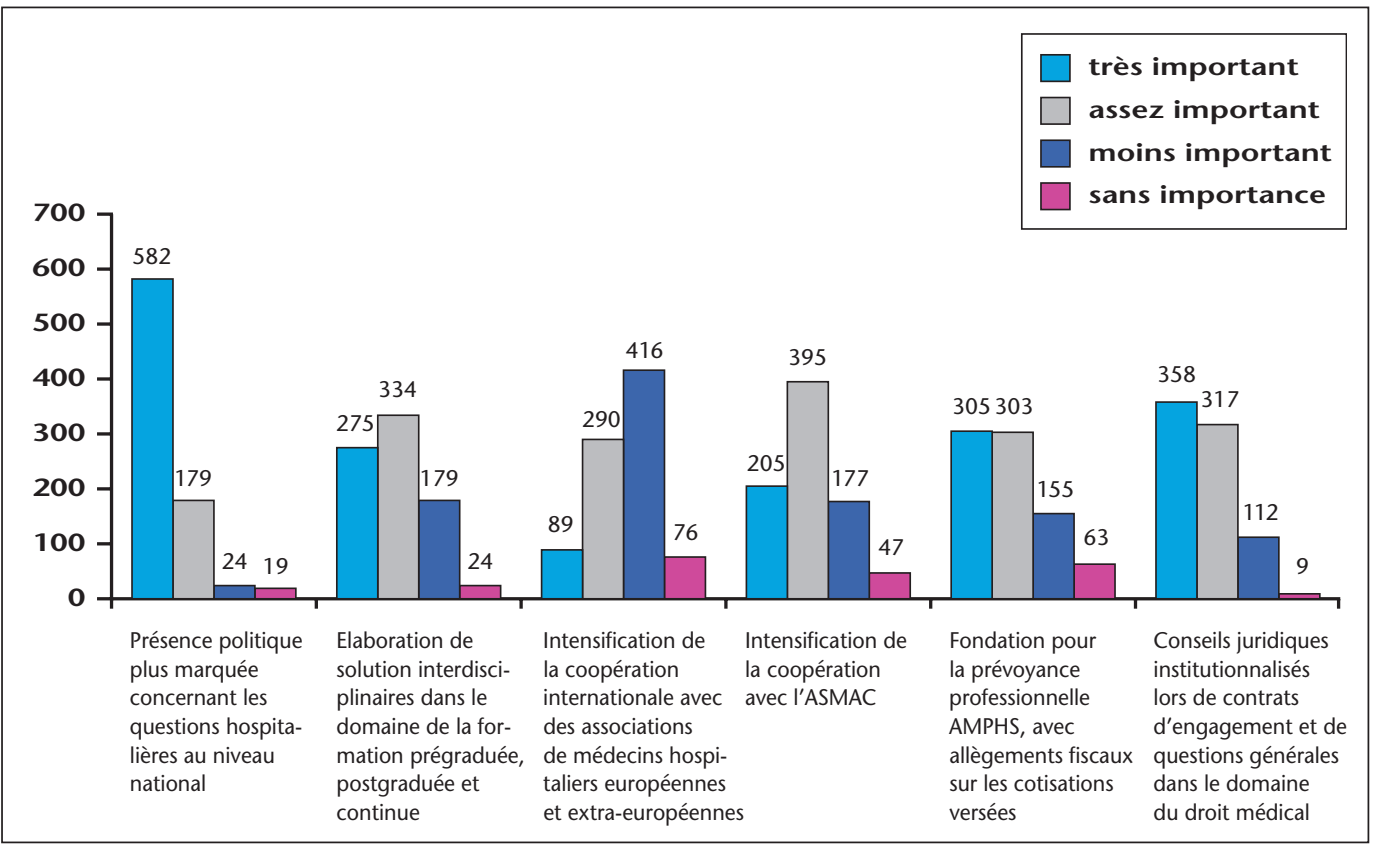

La figure 1 renseigne sur les réponses données aux questions posées.

Selon ce tableau, les médecins-cadres de la base se prononcent majoritairement en faveur de: - présenter une nouvelle proposition à la Chambre médicale en vue d'une reconnaissance en tant qu'organisation de base;

- verser deux cotisations de membre, une cotisation réduite auprès de la société cantonale et la cotisation de membre de l'AMPHS (Fr. 150.- actuellement), pour autant que l'admission de l'AMPHS par la Chambre mé- dicale en tant qu'organisation de base ne puisse se faire qu'à cette condition (affiliation simultanée obligatoire à la société cantonale).

Les médecins-cadres interrogés ont répondu diversement à la question de savoir si un nouveau refus de la demande d'admission à l'AMPHS en tant qu'organisation de base les inciterait à sortir de la FMH.

- 375 des personnes interrogées (env. 46,6\%) ne voudraient pas sortir de la FMH; 
- 39 personnes seulement (env. 4,9\%) démissionneraient sûrement de la FMH;

- cela dit, 309 des personnes interrogées (env. $38,4 \%$ ) sortiraient éventuellement de la FMH;

- et 107 (env. 13,3\%) sont encore indécises.

Remarque au sujet des pourcentages: même s'il n'était pas demandé de citer plusieurs réponses, plusieurs personnes ont donné des réponses multiples qui ont malgré tout été évaluées, ce qui explique un total de 830 réponses pour 804 participants.

\section{Enquête concernant les activités et les prestations de l'AMPHS}

Nous avons saisi l'occasion, dans une seconde partie, de demander aux médecins-cadres de la base quelles sont les activités et les prestations qu'ils considèrent comme les plus importantes. La figure 2 renseigne sur les résultats obtenus.

De l'avis des médecins interrogés, la présence politique plus marquée concernant les questions hospitalières dans toute la Suisse apparaît donc comme très importante (582 citations, à savoir $72,3 \%)$, ainsi que l'offre en conseils juridiques institutionnalisés (358 citations, à savoir env. 47\%).

Les éléments suivants sont considérés comme assez importants:

- la collaboration avec l'ASMAC (395);

- l'élaboration de solutions interdisciplinaires dans le domaine de la formation prégraduée, postgraduée et continue (334);

- la fondation de prévoyance professionnelle de l'AMPHS, avec allègements fiscaux sur les cotisations versées (303).

La coopération internationale a été majoritairement perçue comme étant moins importante (416 citations).

\section{Conclusions provisoires}

Il n'est pas question ici, de lancer une polémique. Il s'agit bien plutôt de définir, à l'aide de l'enquête menée auprès de la base, une certaine direction de notre politique pour ces prochaines années. Nos membres ne semblent pas être insatisfaits de la Fédération des médecins suisses au point de vouloir la quitter à grands fracas. Toutefois, lorsqu'il est question de l'affiliation aux sociétés cantonales de médecine, les résultats pourraient bien donner, ici et là du moins, une toute autre image.
Il va donc de soi que dans sa politique, l'AMPHS doit elle aussi choisir la voie de la coopération et du consensus si elle veut une présence du corps médical «au front» reposant sur des assises les plus solides possibles. La condition pour ce faire est que, lors de futurs débats de politique structurelle au sein de la FMH, les médecins-chef et les médecins adjoints se voient donner, dans les structures de la fédération, le poids qui leur revient et correspond à leurs responsabilités en matière de gestion des ressources du système de santé. Même si l'on ne saurait accorder un crédit absolu aux statistiques, les chiffres de celles-ci montrent à tout le moins un malaise de la part des médecins dirigeants d'hôpitaux au sein des structures existantes.

Il semble que nos membres reconnaissent également la nécessité d'un rapprochement avec un autre groupe de médecins hospitaliers, celui des médecins-assistants et chefs de clinique. Les conditions générales plus rigoureuses qui frappent les assistants et chefs de clinique de par la nouvelle loi sur le travail demandent une structuration beaucoup plus claire des temps de travail et de formation au sein d'un hôpital, afin de ne pas compromettre, malgré la somme de travail immense à accomplir dans le secteur des prestations de soins, la formation postgraduée de médecins spécialistes compétents par de nouvelles chicanes administratives.

Par la même occasion, notons que les efforts fournis par les médecins d'hôpitaux dans leur ensemble (médecins-chefs, médecins adjoints, chefs de clinique et médecins-assistants) doivent tendre à un déroulement du travail le plus rationnel et le plus performant possible. Il s'agit aussi de relever que les instances politiques ne sauraient ainsi se soustraire à leurs responsabilités, sous le fallacieux prétexte de mesures organisationnelles. Viser à disposer de médecins hautement qualifiés dans le domaine de la santé publique est une responsabilité essentielle, pour les instances politiques également: un oui franc et massif à la Loi sur le travail doit donc s'accompagner de la mise à disposition des ressources éventuellement nécessaires.

Le besoin croissant de sécurité sociale dans le domaine de la prévoyance-vieillesse qui se manifeste chez nos membres est certainement à mettre en relation avec leur situation financière devenue plus difficile. Un souhait des plus légitimes qu'il s'agira de prendre mieux en compte à l'avenir. 